\title{
Mulching é uma opção para o aumento de produtividade da melancia
}

\author{
Ricardo Alexandre Lambert ${ }^{1}$, Leonardo Silva Barro ${ }^{1}$, Karoeynne Silva Gomes do Carmo ${ }^{1}$, \\ Aldaisa Martins da Silva de Oliveira ${ }^{1}$, Andréia Aparecida Borges ${ }^{2}$
}

\author{
${ }^{1}$ Instituto Luterano de Ensino Superior de Itumbiara - ILES/ULBRA, Itumbiara, Goiás, Brasil. E-mail: \\ ricardolambert1981@hotmail.com, leonardo.barro@yahoo.com, karoeynnegomes@gmail.com, aldaisa_itb@hotmail.com \\ ${ }^{2}$ Faculdade Santa Rita de Cassia, Itumbiara, Goiás, Brasil. E-mail: andreiaa_borges@ hotmail.com
}

Recebido: 14/07/2016; Aceito: 26/11/2016

\section{RESUMO}

A cobertura do solo com filme plástico ("mulching”) é uma prática agrícola que visa principalmente reduzir a evaporação da água e o aparecimento de plantas daninhas, além de diminuir o uso de herbicida, impedir o contato de frutos e folhas com o solo. Estes benefícios no sistema de produção de melancia podem resultar em menor custo, maior produtividade e qualidade dos frutos produzidos. Neste contexto, objetivou-se com este trabalho avaliar o desenvolvimento, a produtividade e a viabilidade econômica do cultivo de melancia com e sem mulching. O experimento foi conduzido na Estação Experimental do ILES/ULBRA em Itumbiara-GO, utilizandose a cultivar "Crimson Sweet". O delineamento experimental utilizado foi o inteiramente casualizado, com dois tratamentos e 16 repetições, sendo um dos tratamentos o cultivo com o mulching plástico e o outro sem a cobertura do solo, totalizando 32 parcelas. Foram avaliadas as seguintes variáveis: produtividade (PRO), número de internódios (NI), comprimento de ramas (CR) e o custo/benefício de produção na região sul de Goiás. Os resultados obtidos evidenciaram que o uso do mulching plástico melhorou todas as características da cultura da melancia em relação ao cultivo sem a cobertura do solo, mostrando ser uma técnica economicamente viável para a produção de melancia na região sul de Goiás.

Palavras-chave: cobertura do solo, aspectos reprodutivos, viabilidade econômica.

\section{Mulching is an option for increasing watermelon yield}

\begin{abstract}
Mulching is an agricultural practice that mainly aims to reduce the evaporation of water and the appearance of weeds, as well as to reduce the use of herbicide, to prevent the contact of fruits and leaves with the soil. These benefits in the watermelon production system can result in lower costs, higher productivity and quality of fruits produced. In this context, the objective of this work was to evaluate the development, productivity and economic viability of watermelon cultivation with and without mulching. The experiment was conducted at the Experimental Station of ILES/ULBRA, in Itumbiara, GO, Brazil, using the cultivar "Crimson Sweet". The experimental design was completely randomized, with two treatments and 16 replications, one of the treatments being the mulching and the other without soil cover, totaling 32 plots. The following variables were evaluated: productivity (PRO), number of internodes (NI), length of branches (LB) and cost/benefit of watermelon production in the southern region of Goiás, Brazil. The results showed that the use of plastic mulching improved All the characteristics of the watermelon culture in relation to the crop without soil cover, showing to be an economically viable technique for the production of watermelon in the southern region of Goiás.
\end{abstract}

Key words: soil cover, reproductive aspects, economic viability. 


\section{Introdução}

A melancia [Citrullus lanatus (Thunb.) Matsum. \& Naki] é uma Cucurbitáceae de grande expressão econômica e social, possuindo propriedades nutricionais, sendo uma fonte de sais e minerais e terapêuticas que aumentam o interesse do consumidor pelo seu fruto (DANTAS et al., 2013).

É originária das regiões quentes da África, considerada uma planta cosmopolita, sendo introduzida no Brasil pelos escravos, que semeavam no meio do cultivo do milho, onde após a guerra civil americana alguns cultivares melhoradas foram introduzidas pelos produtores em São Paulo (FILGUEIRA, 2000).

A cultura possui um crescimento estoloníferos denominadas ramas que podem alcançar até 5 metros, possuindo limbos profundamente recortados, gavinhas para melhor fixação da mesma ao solo, o seu sistema radicular é extenso no sentido mais superficial do solo horizontalmente na camada até 30 centímetros, possuindo flores femininas e masculinas necessitando de polinização de insetos para reprodução.

A produção de melancia no Brasil, está em torno de 2 milhões de toneladas, possuindo o Rio Grande do Sul como maior produtor com 343 mil toneladas, seguido por Goiás com 273 mil toneladas e a Bahia com 260 mil toneladas (ANDRADE JUNIOR et al., 2007). Na região nordeste essa olericultura é de grande valor econômico para pequenos agricultores por ser uma cultura de fácil manejo e menor custo de produção (ANDRADE JÚNIOR et al., 2007).

Atualmente, a busca por tecnologias que visam o aumento da produtividade e a redução custo é crescente, principalmente, devido à escassez da mão de obra. Os agricultores estão em uma busca constante por práticas agrícolas que viabilizam o seu empreendimento, melhorando a interação entre planta, ambiente e práticas fitossanitárias que possibilita uma melhor qualidade de frutos, e dentre essas práticas o uso de cobertura do solo, a utilização de sementes melhoradas, irrigação e o uso racional de defensivos agrícolas estão entre as melhores opções para melhorar a produção de melancia.

A cobertura do solo ("mulching") é uma prática utilizado há muitos anos no país. Inicialmente, esta prática foi utilizada nas culturas de morango e abacaxi, mas, atualmente, vem se expandindo para a cultura da melancia, uma vez que, o uso possibilita um aumento significativo na produtividade e qualidade de frutos, além de melhorar o microclima do solo por alterar o saldo da radiação na superfície, evitando assim a evaporação da água, diminuindo as irrigações e incidências de doenças foliares (LIMA JUNIOR; LOPES, 2009).

Vários materiais podem ser utilizados para cobrir o solo, sendo classificados em orgânicos como resíduos de culturas (palha, folhas, serragens) e os inorgânicos como cascalhos e plásticos. Entre os plásticos, temos o polietileno preto-branco, preto-prata e preto-preto, a escolha pelo melhor, dependerá da época do ano e condições climáticas do local a ser aplicado. O maior problema dessa técnica é a falta de conhecimento dos agricultores para aplicar o mulching e controlar as irrigações na cultura.

Devido a carência de estudos que apresentem resultados do custo benefício da aplicação do mulching na cultura da melancia, o objetivo desse trabalho foi avaliar o desenvolvimento, a produtividade e a viabilidade econômica do cultivo de melancia cultivar Crimson Sweet, com e sem mulching plástico pretobranco.

\section{Material e Métodos}

O trabalho foi conduzido na Estação Experimental do ILES/ULBRA em Itumbiara-GO, com altitude média de $488 \mathrm{~m}$, definido pelas coordenadas geográficas de $18^{\circ} 40^{\prime} 97^{\prime \prime}$ latitude Sul e 49 $19^{\prime} 19^{\prime \prime}$ longitude Oeste. De acordo com a classificação de INMET (2014), o clima na região enquadra-se no tipo $\mathrm{AW}$, característico dos climas úmidos tropicais, com duas estações bem definidas, seca no inverno e úmida no verão, com precipitação média anual entre 1200 e $1800 \mathrm{~mm}$. O solo foi classificado como Latossolo Vermelho distrófico. Antes da instalação do experimento foi coletada amostra de solo na profundidade de $0-0,20 \mathrm{~m}$ e as principais propriedades químicas do solo são mostradas na Tabela 1.

$\mathrm{O}$ delineamento experimental utilizado foi $\mathrm{o}$ inteiramente casualizado (DIC), com dois tratamentos e dezesseis repetições. Os tratamentos foram constituídos do cultivo convencional de melancia sem cobertura no solo e o outro com a cobertura do solo com mulching plástico preto/branco.

Tabela 1. Resultado da análise química do solo. Itumbiara, 2016.

\begin{tabular}{|c|c|c|c|c|c|c|c|c|c|}
\hline Textura & $\mathrm{pH}$ & $\mathrm{P}$ & $\mathrm{K}$ & $\mathrm{Ca}$ & $\mathrm{Mg}$ & $\mathrm{Al} \mathrm{e} \mathrm{H}$ & $\mathrm{V}$ & CTC pH 7,0 & SB \\
\hline & $\mathrm{CaCl}_{2}$ & $---m$ & 3 ---- & & $-\mathrm{cmc}$ & 3------ & $\%$ & ------- cmol & ---- \\
\hline Argilosa & 5,30 & 13,54 & 140 & 5,78 & 1,88 & 6,48 & 55,30 & 14,40 & 8,02 \\
\hline
\end{tabular}


A adubação mineral foi realizada de acordo com as análises do solo e necessidade da cultura como recomendado por Ribeiro et al. (1999). A dose aplicação no sulco de plantio foi de $770 \mathrm{~kg} \mathrm{ha}^{-1}$ da formulação 0420-20 (NPK). A adubação de cobertura foi realizada com a aplicação da formulação 20-00-20 (NPK), aos 25 e 45 dias após a emergência das plantas.

Os tratos fitossanitários foram realizados com a aplicação de defensivos agrícolas e as dosagens acompanharam as recomendações dos produtos registradas para cultura, via pulverizador costal.

A implantação do experimento ocorreu no dia 25 de fevereiro de 2016, no sistema de plantio convencional, mediante a utilização de gradagem e encanteirador, dividindo a área em 32 parcelas iguais de 3,00 m de largura por 1,50 $\mathrm{m}$ de comprimento, com uma área total da parcela de $4,50 \mathrm{~m}^{2}$ possuindo uma área útil de 2,40 $\mathrm{m}^{2}$ a ser avaliada. A área total do plantio foi de $216 \mathrm{~m}^{2}$, sendo conduzido em sistema de irrigação por gotejamento, com três irrigações semanais no mulching e cinco no cultivo convencional, variando a lâmina de acordo com as condições climáticas.

O espaçamento de plantio foi de 3,00 m entre sulco e $0,80 \mathrm{~m}$ entre plantas. A semeadura foi manual, em uma profundidade de $3 \mathrm{~cm}$, o desbaste foi feito aos 25 dias deixando quatro plantas por parcela, descartando a linha de bordadura e avaliando somente a parte interna das parcelas, ou seja, apenas 2 plantas por parcela.

Após o ciclo da cultura foi avaliado o desempenho agronômico da melancia, onde para avaliar a produtividade, foram colhidos todos os frutos da parcela e pesados em uma balança digital, tendo seu resultado em quilos por hectare. O número de internódios foi contado a partir da primeira flor feminina e totalizados por parcela. O comprimento de ramas foi medido com uma trena de fita com o comprimento de 30 metros apresentados em metros nos resultados e em relação ao custo benefício da utilização do mulching plástico, foram levantados os custos de produção e comparados os resultados.

Os dados obtidos foram submetidos ao teste de $\mathrm{F}$ por meio da análise de variância, e as médias foram comparadas pelo teste de Tukey a 5\% de probabilidade, analisados estatisticamente pelo o software Sisvar, versão 5.3 (FERREIRA, 2014).

\section{Resultados e Discussão}

Os resultados da análise de variância constataram que houve diferenças significativas para o cultivo de melancia com mulching plástico em relação ao cultivo convencional sem cobertura no solo, para as variáveis produtividade, número de internódios e comprimento de ramas (Tabela 2).
$\mathrm{Na}$ Tabela 2, é possível observar na análise de variância, que houve diferença significativa a $1 \%$ nos parâmetros de produtividade e comprimento de ramas e a 5\% no parâmetro de número de internódios da melancia cultivada com e sem o mulching plástico. Quando comparadas suas médias pelo teste de Tukey a $5 \%$, foi possível identificar que em todos os parâmetros avaliados, o cultivo no mulching se destacou em relação ao cultivo convencional (Tabela 3 ).

Resultados semelhantes foram obtidos por Serafim et al. (2015), onde combinou diferentes tipos de mulching e coberturas agrotêxtil, possibilitando uma maior produtividade em relação ao cultivo convencional, confirmando os resultados do presente trabalho. Em relação ao resultado obtido dos parâmetros números de internódios por parcela e comprimento de ramas também foram confirmados pelo trabalho desenvolvido por Silva et al. (2014), onde o mesmo avaliou os aspectos vegetativos e reprodutivos da melancia, submetidos ao cultivo do mulching e diferentes lâminas de irrigação, obtendo um melhor desempenho do mulching em relação ao cultivo convencional. Outro fator importante que devemos destacar ao se quantificar o número de internódios por plantas seria sua relação com a quantidade de frutos por planta, uma vez que, quanto maior for esse número produzido pelas plantas, maior será a quantidade de inflorescências femininas e masculinas, possibilitando maior quantidade de frutos.

Tabela 2. Resultados da análise de variância para produtividade (PRO), número de internódios por parcela (NI), e comprimento de ramas (CR), no experimento efeito do uso do mulching plástico sobre aspectos agronômicos da melancia (Citrullus lanatus (Thunb.) Matsum \& Nakai) cultivada com e sem cobertura do solo. Itumbiara-GO, 2016.

\begin{tabular}{lcccc}
\hline \multirow{2}{*}{$\begin{array}{l}\text { Fonte } \\
\text { variação }\end{array}$} & \multirow{2}{*}{ de } & \multicolumn{3}{c}{ Quadrados médios } \\
\cline { 3 - 5 } & & PRO $\left(\mathrm{t} \mathrm{ha}^{-1}\right)$ & $\mathrm{NI}(\mathrm{und})$ & $\mathrm{CR}(\mathrm{m})$ \\
\hline Tratamentos & 1 & $1132,523^{* *}$ & $325,125^{*}$ & $21,780^{* *}$ \\
Resíduo & 30 & 28,519 & 58,212 & 0,470 \\
\hline CV $(\%)$ & - & 24,59 & 21,27 & 20,21 \\
Média & - & 21,75 & 35,87 & 3,39 \\
\hline * e**-significativos 5\% e 1\% de probabilidade pelo teste $\mathrm{F}$, respectivamente.
\end{tabular}

Tabela 3. Valores médios de produtividade (PRO), número de internódios por parcela (NI), e comprimento de ramas (CR), no experimento efeito do uso do mulching plástico sobre aspectos agronômicos da melancia (Citrullus lanatus (Thunb.) Matsum. \& Nakai) cultivada com e sem cobertura do solo. Itumbiara-GO, 2016.

\begin{tabular}{llll}
\hline TRATAMENTOS & PRO $\left(\mathrm{t} \mathrm{ha}^{-1}\right)$ & NI (und) & CR (m) \\
\hline Mulching & $27,664 \mathrm{a}$ & $39,062 \mathrm{a}$ & $4,218 \mathrm{a}$ \\
Convencional & $15,766 \mathrm{~b}$ & $32,687 \mathrm{~b}$ & $2,568 \mathrm{~b}$ \\
\hline DMS & 3,85 & 5,509 & 0,495 \\
\hline
\end{tabular}

*Médias seguidas da mesma letra, na coluna, não diferem estatisticamente entre si pelo teste de Tukey a 5\% de probabilidade. 
Silva et al. (2014) cita os autores Caron e Heldwein (2000) e Teófilo (2009), que confirmam que o mulching plástico aumenta a proteção da planta, funcionando como uma barreira física controlando plantas daninhas e diminuindo o consumo de água por ciclo da cultura. Outro aspecto importante em relação à quantidade de internódios, que se deve destacar é que quanto maior for seu número, maior será a quantidade de inflorescências tanto masculina quanto feminina, possibilitando maiores chances de geração de frutos.

$\mathrm{Na}$ análise feita para identificar o custo benefício do cultivo da melancia, nenhum tipo de cultivo demonstrou ser inviável, uma vez que suas receitas foram maiores que seus custos, destacando o cultivo no mulching plástico, o melhor tratamento, possibilitando uma margem de segurança ao produtor de $53 \%$, onde a cada $\mathrm{R} \$ 1,00$ (um real) investido seu retorno é de $\mathrm{R} \$ 1,81$ (um real e oitenta e um centavo), mesmo com um custo mais alto, seu retorno é maior, o que torna o investimento lucrativo.

Resultados de viabilidade de cultivo da melancia foram feitos por Assunção et al. (2014) nos municípios de Goiatuba e Morrinhos, que confirmaram que o investimento do cultivo da melancia no sul do estado de Goiás é viável, se tornando uma opção para cultivos de safrinha no estado cultivo da melancia no sul do estado de Goiás é viável, se tornando uma opção para cultivos de safrinha no estado.

Tabela 4. Comparativo de custo médio da produção da melancia em diferentes tipos de cultivo em Itumbiara-GO, 2016.

\begin{tabular}{|c|c|c|c|c|c|c|}
\hline Tipo de cultivo & $\begin{array}{c}\text { Produção } \\
\mathrm{kg} / \mathrm{ha} / \mathrm{ano} \\
\text { (A) }\end{array}$ & $\begin{array}{c}\text { Margem da } \\
\text { produção } \mathrm{R} \$ / \text { ha } \\
\text { (B) }\end{array}$ & $\begin{array}{c}\text { Custo total } \\
\mathrm{R} \$ / \mathrm{ha} \\
\text { (C) }\end{array}$ & $\begin{array}{c}\text { Relação } \\
\text { benefício/custo } \\
(\mathrm{B} / \mathrm{C})\end{array}$ & $\begin{array}{l}\text { Ponto de nível. } \\
\text { (C/P) }\end{array}$ & $\begin{array}{c}\text { Margem de } \\
\text { segurança \% } \\
(\mathrm{C}-\mathrm{B} / \mathrm{B})\end{array}$ \\
\hline Mulching & 27.664 & $16.598,40$ & $8.941,20$ & 1,81 & 14.902 & $-0,53$ \\
\hline Convencional & 15.766 & $9.459,60$ & $7.186,20$ & 1,31 & 11.977 & $-0,24$ \\
\hline
\end{tabular}

Obs.: (A) Produtividade média de 1 ha, B) Margem total: preço x quantidade comercial produzida; (C) Custos efetuados para obtenção da produção;

(P) preço médio anual da melancia $\mathrm{R} \$ / \mathrm{Kg}(\mathrm{R} \$ 0,60)$.

\section{Conclusões}

Diante do exposto é possível concluir que, existem diferenças significativas na avaliação da produtividade e nos aspectos vegetativos da melancia no cultivo convencional, quando comparado ao cultivo no mulching plástico, onde o mulching se destacou em todos os parâmetros avaliados como produtividade, número de internódios por parcela, comprimento de ramas e custo/benefício, possuindo uma viabilidade econômica para cultivo na região sul de Goiás.

\section{Referências Bibliográficas}

ANDRADE JUNIOR, A. S.; RODRIGUES, B. H. N; ATHAYDE SOBRINHO, C.; BASTOS, E. A.; MELO, F. B.; CARDOSO, M. J.; SILVA, P. H. S.; DUARTE, R. L. R.A cultura da melancia, Brasília-DF: Embrapa Informação Tecnológica, 2007, 85 p. (Coleção Plantar, 57).

ASSUNÇÃO, P. E. V.; WANDER, A. E.; CARDOSO, J. S. Viabilidade Econômica do Sistema de Produção de Melancia no Sul de Goiás. Goiânia-GO: SEGPLAN/IMB Instituto Mauro Borges de Estatísticas e Estudos Socioeconômicos. Conjuntura Econômica Goiana, Junho $2014 . \quad$ Disponível em: <http://www.imb.go.gov.br/pub/conj/conj29/artigo_03.pdf>. Acesso em 20 fevereiro de 2016.

CARON, B. O.; HELDWEIN, A. B. Consumo d'água e coeficiente de cultura para o meloeiro cultivado em estufa plástica na primavera. Revista Brasileira de Agrometeorologia, Santa Maria, v. 8, n. 1, p. 19-25, 2000.
DANTAS, M. S. M; GRANJEIRO, L. C; MEDEIROS, J. F.; CRUZ, C. A; CUNHA, A. P. A. Rendimento e qualidade de frutos de melancia cultivada sob proteção de agrotêxtil combinado com "mulching" plástico. Revista Brasileira de Engenharia Agrícola e Ambiental, Campina Grande-PB, v.17, n.8, p.824-829, 2013.

FERREIRA, D. F. Sisvar: a Guide for its Bootstrap procedures in multiple comparisons. Ciência e Agrotecnologia, LavrasMG, v.38, n.2, p. 109-112, 2014.

FILGUEIRA, F.A. R. Novo manual de olericultura: agrotecnologia moderna na produção e comercialização de hortaliças. Viçosa-MG: UFV, 2000, 402 p.

INMET. INSTITUTO NACIONAL DE METEOROLOGIA. Estação meteorológica de observação de superfície $\begin{array}{llll}\text { automática. } & 2014 . & \text { Disponível }\end{array}$ <http://www.inmet.gov.br/portal/index.php?r=estacoes/estaco esautomaticas>. Acesso em 21 jun. 2016.

LIMA-JUNIOR, J. A.; LOPES, P. R. A. Avaliação da cobertura do solo e métodos de irrigação na produção de melancia. Semina: Ciências Agrárias, Londrina-PR, v. 30, n. 2, p. 315-322, abr./jun. 2009.

RIBEIRO, A. C.; GUIMARÃES, P. T. G.; ALVAREZ V., V. H. Recomendações para o uso de corretivos e fertilizantes em Minas Gerais: $5^{\text {a }}$ aproximação. Comissão de Fertilidade do solo do Estado de Minas Gerais, Viçosa-MG, 1999. 359 p.

SERAFIM, E. C. S.; GRANGEIRO, L. C.; ESPINOLA SOBRINHO, J.; NEGREIROS, M. Z.; DANTAS, M.S.M. Acúmulo e exportação de nutrientes em melancia cultivada sob proteção de agrotêxtil. Revista Agropecuária Científica 
no Semiárido, Campina Grande-PB, v. 11, n. 1, p. 136-144, 2015.

SILVA, D. R. M.; CUNHA, C. S. M.; FELIPE, E. A. Aspectos vegetativos e reprodutivos para a cultura da melancia sob diferentes coberturas de solo e níveis de irrigação em Teresina - PI. Revista Agropecuária Científica no Semiárido, Campina Grande-PB, v. 10, n. 2, p. 96-103, 2014.
TEÓFILO, T. M. S. Interferência de plantas daninhas no crescimento e na eficiência de uso da água na cultura do meloeiro nos sistemas de plantio direto e convencional. 2009. 80f. Dissertação (Mestrado em Fitotecnia: Área de concentração: Agricultura tropical). UFERSA, Mossoró, 2009. 